\title{
NEXT-GENERATION COLLABORATIVE ROBOTIC SYSTEMS FOR INDUSTRIAL SAFETY AND HEALTH
}

\author{
NIKHIL DESHPANDE, JESÚS ORTIZ, IOANNIS SARAKOGLOU, CLAUDIO SEMINI, NIKOS \\ TSAGARAKIS, ANAIS BRYGO, JORGE FERNANDEZ, MARCO FRIGERIO, LORENZO SACCARES, \\ STEFANO TOXIRI \& DARWIN G. CALDWELL \\ Department of Advanced Robotics, Istituto Italiano di Tecnologia, Genova, Italy
}

\begin{abstract}
Occupational Safety and Health (OSH) is defined through three objectives: (i) maintenance of workers' health; (ii) improvement of working environment and safety; and (iii) promotion of a work culture that supports health and safety. In industry, the most frequent threat to workers' health is musculoskeletal disorders. Furthermore, even routine tasks in certain work environments (nuclear, construction, disaster response, marine, chemical, etc.) expose workers to extreme risks like explosions, contaminations, fires, confined spaces, debris, toxic gases, etc. The goal of this research is to design and develop advanced collaborative robotic technologies towards: (i) reducing workers' physical stress and improving their health through a novel modular full-body wearable exoskeleton with arm, lower-back, and leg modules, allowing full motion dexterity; and (ii) avoiding hazard-prone worker environments and improving safety through a new collaborative master-slave teleoperation system consisting of: (a) a hydraulicallydriven, quadruped field robot with a robotic manipulator arm for operation in hazardous environments; (b) a hand exoskeleton master device for teleoperation control. The article presents the current status of development of these technologies. Preliminary validation of the exoskeleton shows reductions in muscular efforts of up to $30 \%$ for the lower back. For the master-slave collaborative system, (a) the Hydraulic Quadruped robot prototypes can traverse rough environments through capabilities that include stair climbing, walking over obstacles, omni-directional trotting with step reflexes, running, jumping, and self-righting; (b) the 7 degrees-of-freedom (DOF) manipulator arm allows object manipulation in a large workspace with dexterous grasping of up to $160 \mathrm{~N}$ of payload; and (c) the novel HEXOTRAC 3-digit hand exoskeleton provides high-resolution tracking of fingers and provides force feedback for intuitive bilateral teleoperation of robotic manipulators. These next-generation industrial exoskeletons and collaborative teleoperation systems can address the emerging challenges in industrial workers' health and safety.

Keywords: robotic exoskeleton, occupational health, hydraulic quadruped, remote teleoperation, haptic tele-manipulation, dexterous manipulator, occupational safety, hand exoskeleton.
\end{abstract}

\section{INTRODUCTION}

The Occupational Safety and Health (OSH) paradigm recognizes the direct and indirect consequences, both human and economic, of work-related accidents and diseases. Successful OSH practices, especially in labour-intensive and hazardous work environments, address issues ranging from industrial hygiene, engineering safety, ergonomics, to toxicity, radiation, potential for explosions, etc. OSH therefore has a strong focus on primarily preventing hazards at the workplace. The World Health Organization defines OSH through three objectives: (i) maintenance of workers' health; (ii) improvement of working environment; and (iii) promotion of a work culture that supports health and safety [1]. These objectives manifest themselves in the everyday concerns of workers' well-being. The definition identifies "safety" and "health" as two separate classes of issues. This implies that efforts in OSH have to address health and safety issues both individually and concurrently.

In the industrial environment, in spite of the on-going trend in automation, many workers are still exposed to physical workloads and environments, which put their health and safety at risk. Within the occupational health perspective, the most frequent threat to workers is 
musculoskeletal disorders (MSDs) due to material handling ( $>30 \%$ of the worker population in the EU), repetitive movements (63\%) and awkward body postures (46\%) [2]. About 44 million EU workers are affected by MSDs at a total annual cost in excess of $€ 240$ billion, or circa $2 \%$ of GDP, to the European economy [3]. Work-related lower back pain and injuries are the most common MSDs $-30 \%$ of European workers reported that their work causes back problems [4].

From the safety perspective, even routine tasks in certain work environments expose workers to extreme risks, such as: (i) nuclear (explosions, contamination, fires, etc.), (ii) chemical, petro-chemical, and iron and steel (biological risks, toxic gases and fumes, fires, etc.), (iii) construction and demolition (air quality, confined spaces, dangerous heights, etc.), (iv) marine and underwater operations (drowning, lack of oxygen, toxic gases, etc.). Hazards may be present in the form of toxicity, radiation, dangers to potential explosions, exposures to chemicals, etc., where the magnitudes reach a point that human exposure would either represent a direct threat to life or long-term health consequences. In the UK alone, around 13,000 deaths occur each year from work-related lung disease and cancer attributed to past exposure, primarily to chemicals and dust at work [5]. In 2014, there were about 3.2 million non-fatal accidents and 3,739 fatal accidents in Europe [3].

\subsection{Robotics in the industrial environment}

Robotic devices in the industrial workplace have been considered as a means of assisting workers' capacity in industrial tasks [6], as well as substituting them and protecting them from exposure to hazardous substances, environments and/or physical agents [5]. In space, defence, security, and nuclear industry, autonomous robots are useful in replacing human workers performing dirty, dull, or unsafe tasks, thus reducing physical, ergonomic and psycho-social risks [7]-[8]. For instance, robots were able to reduce dust exposures by $75 \%$ in a plastic-moulding operation [5].

Nevertheless, in modern production facilities with high product mixes and dynamic manufacturing, the human capacity to be flexible and adaptable is still beneficial [6]. A better approach therefore, is to combine the optimal advantages of robotics with the optimal skills, creativity, decision-making, flexibility, and adaptability of humans. Furthermore, operations in hazardous environments necessarily involve humans-in-the-loop. The perception and action capabilities of the systems mimicking human capabilities significantly improve the transparency and stability of interactions between the operator and the environment.

\subsection{Contributions of this research}

It is therefore, essential to address occupational health and occupational safety independently as well as comprehensively. The goal of this research is to design and develop collaborative robotic technologies dedicated to improving the condition of health and safety in industrial applications.

Improvement in occupational health on the factory floor is proposed through assistance, i.e., aiding the worker in their tasks through a wearable, modular, full-body exoskeleton. The design, consisting of arm and lower back modules, is focused on flexibility (adaptability to different tasks) and comfort, fully profiting from the combination of human flexibility and the assistance in tasks through active control.

Enhanced occupational safety in hazardous environments is proposed through substitution, i.e., removing the worker from the unsafe area and having robotic technologies do the same tasks through master-slave teleoperation. To achieve this goal, a collaborative 
robotic teleoperation system is proposed, in two parts: (i) a slave field robot composed of a hydraulically-driven, quadruped field robot, attached with a dexterous manipulator arm for operation in unstructured, hazardous environments; and (ii) a master station consisting of a haptic hand exoskeleton and joystick master device for the teleoperation of the field robot and manipulator arm.

In this article, the status of development of these individual technologies is presented, including the design concepts, fabrication, and implementations. The functionality of the individual technologies is validated through relevant task-based trials including, load lifting using exoskeleton, locomotion capabilities of the quadruped robot, tracking accuracy and haptic capabilities of the hand exoskeleton master, and high payload manipulation with the robotic arm.

\section{DESIGN OF THE WEARABLE, FULL-BODY, MODULAR EXOSKELETON}

The main objective of an industrial exoskeleton is the reduction of the muscular efforts of the operator during manual handling tasks. For instance, lifting and lowering operations require an assistive device for the lower back of the user. Some other industrial applications require postural support, where the operator is forced to adopt awkward positions. In such cases, a modular exoskeleton design allows the system to adapt to the application.

In an industrial setting, the exoskeleton is a daily use equipment. Therefore, its acceptability by the operators is a key design motivation. This article presents Robo-Mate, a modular, wearable exoskeleton with trunk and arm modules, whose design had the following key motivating factors:

- Comfort: Two main aspects affect the comfortability of an exoskeleton: the weight and the body attachments. Extending the exoskeleton to the ground counters the weight, but increases the design complexity significantly and the inertia thereby reducing the transparency of the system. The Robo-Mate exoskeleton targets low-to-medium loads (between 5 and $15 \mathrm{Kg}$ ), which allows the possibility of a simpler design, without ground support. The body attachments, where the forces between the exoskeleton and the operator are exchanged, are designed in such a way as to be efficient in force transmission and not cause discomfort to the user.

- Transparency: The system transparency is dictated by the kinematics of the design and the back-driveability of the actuation. To avoid any movement restrictions, unactuated joints are used to provide support against gravity. Using force control with actuated and unactuated joints, a transparent control is implemented allowing free movement when the assistive forces are not active.

- Intuitive control: In natural operation, the operator intuitively works in a similar way, with or without the exoskeleton. Therefore, the system should follow the movement of the operator, providing the correct level of assistive forces. The RoboMate exoskeleton work on the identification of user intention and the knowledge of external conditions, e.g., load weight, to implement intuitive control for the joints.

\subsection{Trunk module}

Spinal muscles are the main contributors to the large lumbar compression forces that cause lower back injuries. The main purpose of the trunk module is the reduction of these forces [9]. The system provides an assistive torque at the hip level, which is transmitted to the body as a pushing force on the upper leg and a pulling force at the upper body (chest and shoulders). Fig. 1 shows the final prototype of the Robo-Mate trunk module, with the corresponding 


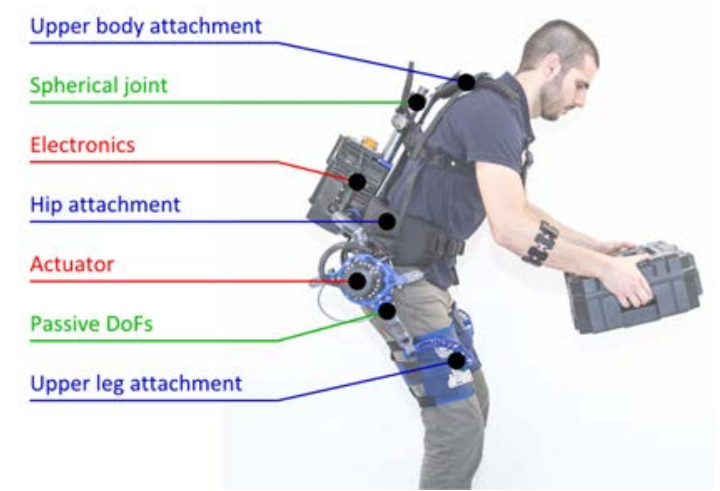

Figure 1: The Robo-Mate exoskeleton with its constituent modules.

attachment points and actuator placement. To allow the freedom of movement to the operator, the system includes a number of passive DOFs in the leg and torso segments. The electronics and control system are placed on the back of the system. Each actuator includes a torque sensor and a magnetic encoder used for the low-level torque control. The electronic box includes an IMU to measure the torso-bending angle, which is used for the high-level control.

\subsection{Arm module}

Due to the complexity of the human shoulder, the arm modules follow a nonanthropomorphic design. The arm modules are mounted on the back of the user and transmit the forces directly to the wrist of the operator. Each arm is composed of two four-bar mechanisms, which allows the vertical displacement, as is commonly seen in the commercial steady-cam mechanisms. The details of the design are found in [10]. The passive version of the arm uses a spring mechanism to compensate for gravity. The preload of the springs is adjusted manually to adapt the system to different loads. Without actuation, the system is more robust, but is only applicable to tasks where the weight of the load is constant. On the other hand, the active arm module substitutes the springs with an actuator and a cable driven mechanism. This increases the flexibility of the system, but makes it more complex, requiring electronics and control [10].

\section{DEVELOPMENT OF THE COLLABORATIVE ROBOTIC TECHNOLOGIES}

As stated earlier, the collaborative teleoperation master-slave system is envisaged for substitution in industrial applications. This section describes the novel designs and technological developments towards the realization of the teleoperation system: (i) the hydraulically powered quadruped field robot; (ii) a dexterous manipulator arm with endeffector hand; and (iii) a hand exoskeleton tele-manipulation master device.

\subsection{Design of the hydraulic quadruped field robot}

For human substitution in dangerous and dirty tasks, quadruped robots have important advantages over wheeled and tracked vehicles in their ability to operate in highly dynamic, unstructured, outdoor areas, e.g., collapsed buildings, disaster sites, forests, and construction sites. A quadruped is also an inherently stable design and does not demand complex 
balancing control systems, compared to a bipedal robot [11]. Considering the hazardous application settings, the final goal of the field robot is mobility and agility on rough terrain, with dynamic motion capabilities. Therefore, the following design requirements are established towards this goal: (i) strong and rugged mechanical structure that can cope with falls without damage; (ii) ability to get up on its own (self-righting) after falling; (iii) ability to squat to save energy and for easier transport; (iv) agility across terrain and obstacles; and (v) weight limited as a trade-off for payload capacity, agility, and overall size.

The version of the robot presented in this article, called the HyQ2Max, is the result of an evolution in design over the last 7 years [11]. The design choices are based on the original HyQ robot [11], [12], including: (i) lengths of the torso and legs, approximately $1 \mathrm{~m}$ for both; (ii) each leg built with three active DOFs allowing 3D positioning capability for the feet; (iii) a forward/backward configuration of the legs (front knees pointing to hind knees) to allow static stability [11] and (iv) the hydraulic actuation dictated by the requirement of versatility and dynamics of the required motions (walking, jumping, running, etc.). Hydraulic actuators give a high power-to-weight ratio and quick response time as compared to electric or pneumatic actuators [13]. Based on these design choices and specifications, the 12-DOF robot was fabricated as shown in Fig. 2. Each of the four legs has three hydraulically actuated joints: Hip Abduction/Adduction (HAA), Hip Flexion/Extension (HFE), and Knee Flexion/Extension (KFE) joints.

The majority of the structural parts are built in aerospace-grade aluminium alloy (type 7075). Rugged leg shells (upper leg) and tubular structures (torso) protect delicate electronics, sensors, and actuators. Kevlar reinforced fiber composite covers protect the central section of the torso that contains the computers, electric power/safety management, and hydraulic system. The torso and leg structures are built so that the robot is not damaged during falls. The hydraulic system of the robot includes highly integrated leg manifolds that are produced with additive manufacturing of AlSiMg aluminium alloy (direct metal laser sintering) [14]. Each joint features high-resolution absolute encoders (ICHaus/Balluff 19-bit) and custom torque sensors or load cells (Futek LCM 325).

Based on the simulated motions and obtained joint ranges and torque profiles, the actuator types and sizes were determined. The HFE and HAA joints require similar positive and negative torques, while the KFE joint needs to produce large torques in one direction (leg extension) and much smaller torques in the other (leg retraction). Table 1 gives the specifications of the fabricated robot. The control (both torque and position) of the joints is implemented on an on-board computer, which communicates with individual actuator/sensor control boards through real-time EtherCAT.

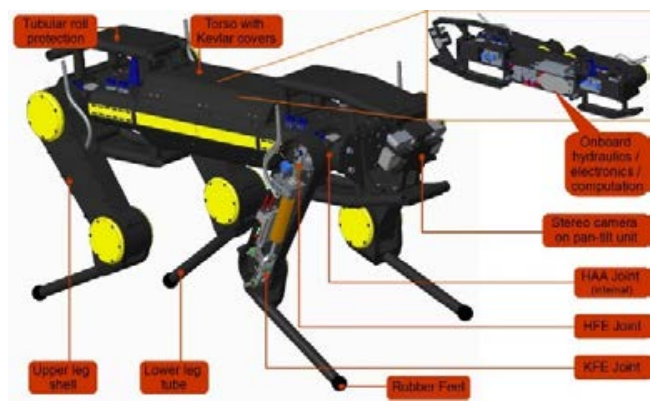

Figure 2: The Hydraulic Quadruped (HyQ2Max) Robot (Inset: cross-section of torso). 
Table 1: System Overview for the HyQ2Max.

\begin{tabular}{|l|l|}
\hline Dimensions & $1.306 \times 0.544 \times 0.918 \mathrm{~m}^{3}$ \\
\hline Link Lengths and weights & $\begin{array}{l}\text { Hip: } 0.117 \mathrm{~m}, 3.54 \mathrm{~kg} \\
\text { Upper leg: } 0.36 \mathrm{~m}, 4.95 \mathrm{~kg} \\
\text { Lower leg: } 0.38 \mathrm{~m}, 1.40 \mathrm{~kg}\end{array}$ \\
\hline Weight & $80 \mathrm{~kg}$ (off-board power) \\
\hline Active DOFs & 12 \\
\hline HAA actuators \& max. torque & Double-vane rotary hydraulic actuators, $120 \mathrm{Nm}$ \\
\hline HFE actuators \& max. torque & Single-vane rotary hydraulic actuators, $245 \mathrm{Nm}$ \\
\hline KFE actuators \& max. torque & $\begin{array}{l}\text { Asymmetric hydraulic cylinders with four-bar linkage, } \\
250 \mathrm{Nm}\end{array}$ \\
\hline Joint range (HAA, HFE, KFE) & $80^{\circ}, 270^{\circ}, 160^{\circ}$ \\
\hline Position sensors & Absolute -262144 cpr, all joints \\
\hline On-board computer & Pentium i5 with real-time Linux @ $1 \mathrm{kHz}$ \\
\hline Controller rate & $1 \mathrm{kHz}$ torque \& position control \\
\hline
\end{tabular}

\subsection{Design of the Robotic Manipulator Arm}

In harsh environments, the field robot should be capable of autonomously performing elementary as well as heavy-duty manipulation tasks, e.g., lifting, carrying loads, or pushing collapsed debris, operating heavy power tools, applying strong forces to open blocked doors, etc. The design of the robotic manipulator arm was therefore, based on powerful manipulation and physical sturdiness objectives to satisfy the above tasks. Additionally, in order to keep a low profile in inertia and mass, actuators with high power-to-weight ratio and large joint range of motion to achieve human-like movement were considered.

The novel design of the robotic manipulator closely resembles an anthropomorphic arrangement - the kinematics includes 3-DOFs at the shoulder, 1-DOF at the elbow, 1-DOF for the forearm rotation and 2-DOFs at the wrist. (Refer Fig. 3). This typical arm configuration provides adequate dexterity as well as redundancy to cope with constraints. The actuation of the arm was based on the integration of seven series elastic actuator units along the kinematic structure of the arm. For the interconnection of the actuator units, the arm design followed an exoskeleton structure approach in which the body of the actuator is floating inside this exoskeleton structure. The actuator is fixed to the structure and the following link using only two flanges located at the same side of the actuator [15].

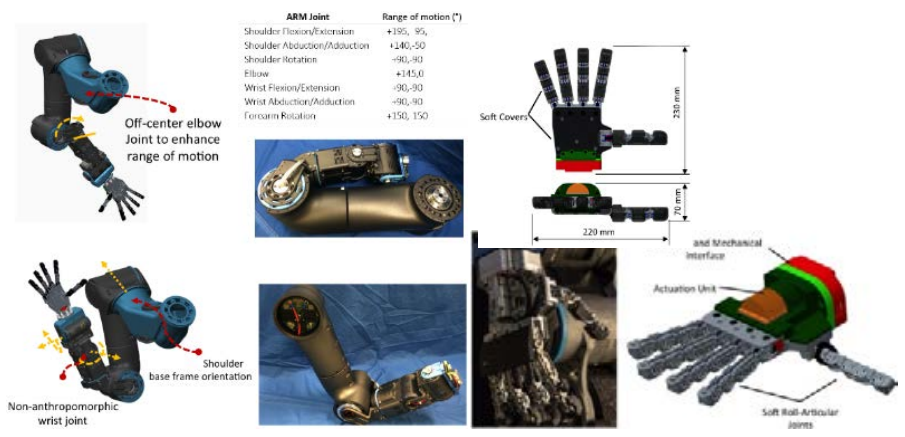

Figure 3: The robotic manipulator arm and the end-effector hand - design and prototype. 
Custom designed actuators (brushless DC motors with harmonic drives and integrated position sensors) power each joint [15]. A 6-DOF force/torque sensor is integrated at the wrist joint, while a camera, embedded at the elbow, provides visualization for the manipulation tasks. A PID position controller controls each joint in a distributed embedded electronic system with one board per joint, running a hard-real-time loop at $1 \mathrm{kHz}$. A central control computer with real-time EtherCAT communication is responsible for task definition, object recognition, and trajectory generation.

\subsubsection{End-effector design}

The end-effector hand is designed with an anthropomorphic shape to adapt to objects, tools, and fixtures. The base design for the end-effector is the Pisa/IIT SoftHand [16], uses a "soft" joints design principle with soft materials and a simplified approach to control of grasping [15]. The hand has 19 DOFs, distributed in an anthropomorphic structure driven by a single actuation unit. The highly under-actuated design transmits power to all joints through a tendon-based structure. The soft joints design, with rolling joints held together by elastic ligaments, allows the hand to withstand impulse events (impacts). A printed soft polymer outer protective shell provides suitable friction for object grasping and manipulation. The hand is actuated by a KollMorgen 30 Watts motor (RBE 00510) with a Harmonic Drive HFUC-8-100 with a reduction ratio of 100:1. The actuation system acts on a Dynema fiber ligament with a diameter of $0.8 \mathrm{~mm}$ and a maximum strength of $1100 \mathrm{~N}$. Fingers, palm, and wrist interfaces are built with high strength aluminium alloy, the electronic boards are placed in the wrist interconnection and protected by an aluminium alloy frame. The total weight is approximatively $1.3 \mathrm{~kg}$.

\subsection{Design of the HEXOTRAC hand exoskeleton}

As noted earlier, hand exoskeletons have the potential to serve as teleoperation devices with optimal structure and feedback forces. In their designs though, the challenge is to embed the actuation and transmission systems in confined spaces, while simultaneously and transparently matching the profound dexterity of the hand without causing a burden. In view of these aspects, a novel, hand exoskeleton design is presented here, based on a 3-digit design, by following the objectives of wear ability, comfort, workspace range, minimal actuation for reduced burden, force feedback, precise finger tracking, and minimal weight. The detailed design specifications and motivations are given in [17].

The design involves a 6-DOF, revolute joints, mechanism for the index-finger, middlefinger and thumb, with a configuration, which can track the position and orientation of the fingertips. The actuation is based on a highly under-actuated mechanism with force feedback transmitted to the distal phalanges of the operator's fingers by providing actuation only to the $1^{\text {st }}$ out of the 6 revolute joints of the serial kinematic chain. The serial linkages are grounded on one side at the base and are attached to the distal phalanges of the fingers through thimbles. This single point of attachment of the links to the fingers allows for quick and easy mounting and removal of the device and keeps the palm, the finger phalanges, and the joints free of mechanical obstructions. The linkage kinematics provide 6-DOF motion (rotations and translations) to the fingertips and allow unobstructed motion of the fingers in their entire workspace. The kinematics of the system support a very large range of hand sizes without having to modify or adapt the linkage lengths.

For finger position tracking, all 6 joints of the exoskeleton linkages are equipped with magnetic position encoders. The $1^{\text {st }}$ joint is measured by the motor encoder with a resolution of 1024 counts per revolution, which after a gear ratio of $30.2: 1$ delivers a resolution of $0.2 \mathrm{e}-$ 


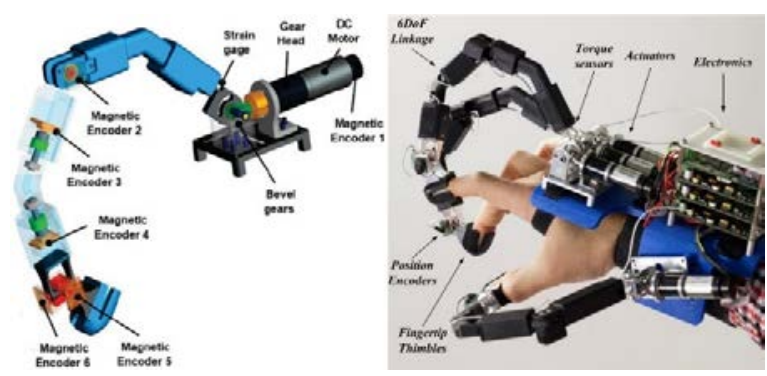

Figure 4: CAD model and prototype of the HEXOTRAC hand exoskeleton.

$3^{c}$ to the first rotary joint. The remaining 5 joints of the exoskeleton are equipped with Austria microsystems AS5045 12-bit rotary magnetic encoders delivering a resolution of $1.533 \mathrm{e}-3^{c}$ at these joints (Refer Fig. 4).

For force feedback, the actuators used in the design have a stall torque of $16 \mathrm{mNm}$, a continuous torque of $5.5 \mathrm{mNm}$, and a gear ratio of $30.2: 1$. The axis of the motor output shaft is rotated by $90^{\circ}$ and torque is transmitted to the $1^{\text {st }}$ joint of the exoskeleton linkage through a pair of high precision anti-backlash bevel gears. For a finger of $10 \mathrm{~cm}$ in length, the available actuator torques correspond to approximately $4.8 \mathrm{~N}$ at stall and $1.66 \mathrm{~N}$ continuous available to a fully extended finger parallel to the horizontal plane. Although these forces are lower compared to grasp and manipulation forces, they are adequate for creating simulations of haptic contacts. Dedicated microcontrollers running $1 \mathrm{kHz}$ control loops control the individual finger interaction forces.

\section{VALIDATION OF THE TECHNOLOGIES}

This section presents the evaluation of the individual technologies in dedicated applicationdependent tasks.

\subsection{Evaluation of the Robo-Mate exoskeleton}

The Robo-Mate exoskeleton was tested in laboratory and industrial environments (Fig. 5):

- Laboratory testing: During these tests, the subject carried out a series of predefined tasks simulating the industrial work. Three tasks were defined: (i) dynamic lifting and lowering of a weight range of 7.5 and $15 \mathrm{Kg}$, (ii) static overhead task with a weight of $2 \mathrm{Kg}$ in one single hand, and (iii) isometric bending task with a trunk flexion of $0,10,20,30,40$ and 50 degrees. The motion of the operator was measured using an optical tracking system. The muscular activity of several muscles (rectus abdominis, erector spinae L3, erector spinae T9, biceps femoris, tibialis anterior, gastrocnemius, and rectus femoris) was recorded using a superficial electromyography system. The arm modules show a reduction in the muscle activity and perceived exertion in the arms. The trunk module indicated a reduction in muscle activity by up to $30 \%$ in lifting tasks, which agrees with earlier results in [6].

- Industrial testing: Qualitative testing was done in simulated industrial environments with separate tasks for each module. The Trunk Module was tested in a cable positioning and fixing task where the operator bends between 30 and 45 degrees in 


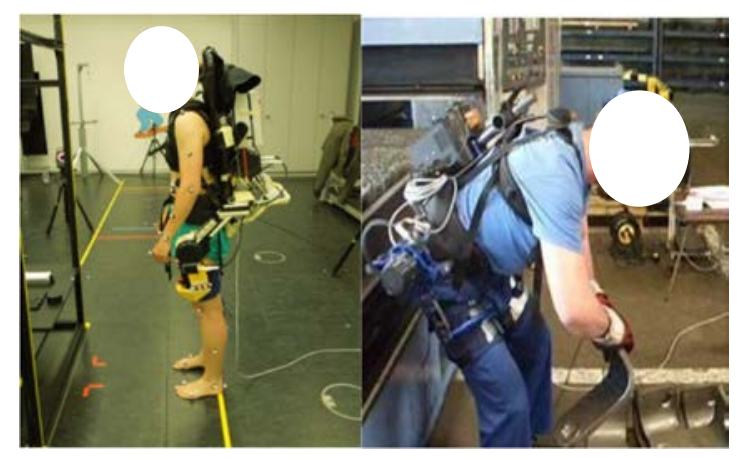

Figure 5: Robo-Mate testing in laboratory (left) and industrial setting (right).

Table 2: Hydraulic Quadruped motions.

\begin{tabular}{|l|c|c|}
\hline Motion & Maximum capability & Reference \\
\hline Walking trot on rough terrain & $0.5 \mathrm{~m} / \mathrm{s}$ & {$[18]$} \\
\hline Walking trot on flat ground & $1.5 \mathrm{~m} / \mathrm{s}$ & {$[18]$} \\
\hline Walking trot with turning & $0.5 \mathrm{~m} / \mathrm{s}$ with $25 \%$ turning & {$[18]$} \\
\hline Crawling on flat ground & $0.1 \mathrm{~m} / \mathrm{s}$ & {$[19]$} \\
\hline Stair climbing & Step height: $0.12 \mathrm{~m} ;$ Step depth: $0.3 \mathrm{~m}$ & {$[20]$} \\
\hline Recovery from lateral push & Perturbation of $500 \mathrm{~N}$ for $1 \mathrm{sec}$ & {$[18]$} \\
\hline
\end{tabular}

a static position. The passive arms were tested in an operation on the car underbody with the hands over the head for the whole cycle. The active arms were tested in two different applications: (i) headlight mounting, and (ii) brake disc fitting. In all the tests, the exoskeleton showed high robustness and importantly, acceptability by the operators without hindrance to movements.

\subsection{Experimental results with the Hydraulic Quadruped robot}

The design of the HyQ2Max robot was aimed at covering a wide range of motions that represent normal and extreme cases in the target environments. A set of motions were defined as important towards validation of the dynamic and agile motion capabilities of the robot. Table 2 summarizes the motion capabilities and the reference papers for their detailed results.

In extreme environments, it is unavoidable that the quadruped robot falls during its operation on challenging terrain. To recover from such falls, the self-righting capability is fundamental for the robot. The robot executes a predefined motion sequence in such situations. Fig. 6 shows the self-righting sequence of HyQ2Max [21]. The maximum torques for the joints on these motions are well within the torques offered by the joint actuators.

\subsection{Preliminary evaluation with the dexterous manipulator arm}

For the evaluation of the robotic manipulator with the dexterous end-effector, it was mounted as part of the IIT humanoid robot, WALK-MAN [22]. The evaluation trials included a heavyduty valve-turning task and a door-opening task. A detailed discussion of the trajectories and control algorithms is beyond the scope of this article and the reader is referred to [22]. A sequence of photographs from the valve-turning task are shown in Fig. 7. 


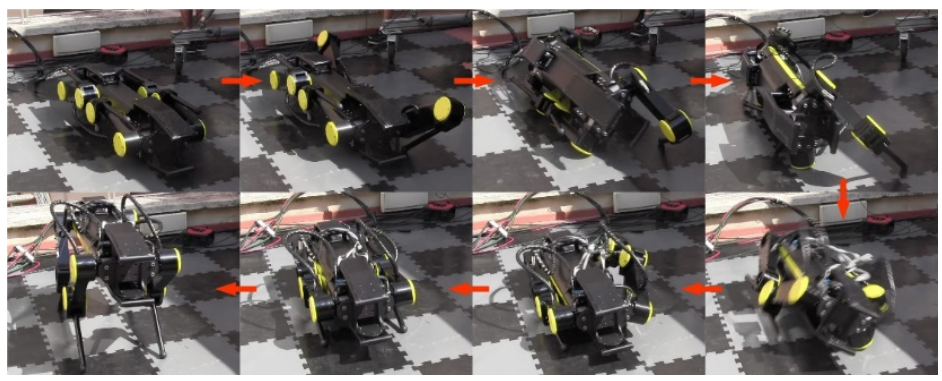

Figure 6: The self-righting motion of the HyQ2Max robot (clockwise from top-left).

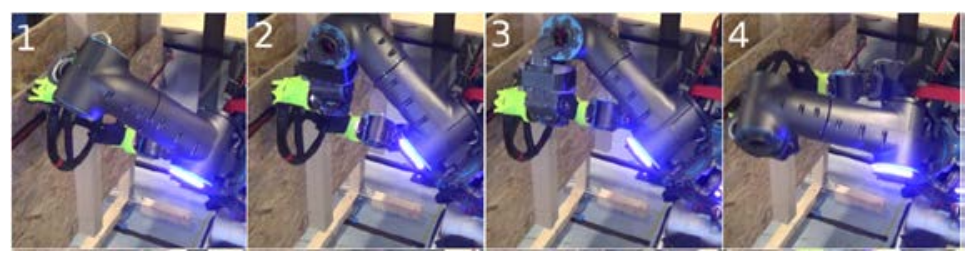

Figure 7: Valve turning task execution with the manipulator arm and end-effector hand [24].

\subsection{Evaluation results with the HEXOTRAC device}

The delivery of force and tactile feedback to the fingertip is highly dependent upon precise tracking of the fingers. Therefore, the proposed HEXOTRAC device includes an integrated tracking system with high resolution and high frame rate. For the evaluation of the tracking, it is noted that the accuracy is a function of the resolution of the encoders and varies within the workspace. Fig. 8 shows the accuracy for the index finger during a natural flexion motion. The markers indicate the 3D position of the fingertip tracked by the exoskeleton. Indicative examples of the accuracy (x, y, z) are shown at 3 points (a), (b) and (c) along the trajectory (in $\mathrm{mm}$ ). The accuracy of the fingertip's orientation tracking is computed in a similar manner. For the flexion trajectory evaluated, the worst-case accuracy corresponding to the 3 axes is presented in Table 3. Additional detailed analysis is given in [17].

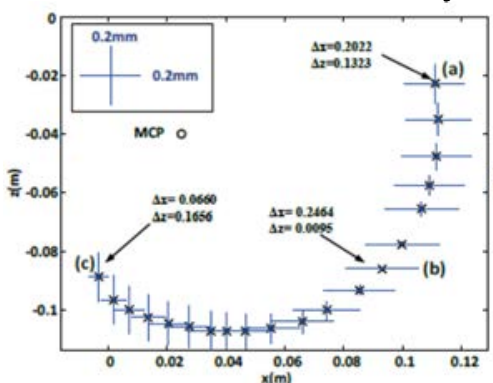

(a)

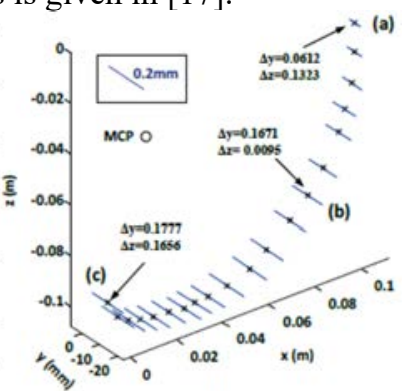

(b)

Figure 8: HEXOTRAC tracking accuracy for a flexion trajectory. (a) $\mathrm{x}$ and $\mathrm{z}$ position; (b) y position. The bars, scaled up to assist visualization, indicate the accuracy. 
Table 3: HEXOTRAC tracking accuracy (worst case).

\begin{tabular}{|l|c|c|}
\hline AXIS & Max. Position Error (mm) & Max. Orientation Error (radians) \\
\hline X & 0.249 & 0.023 \\
\hline Y & 0.190 & 0.031 \\
\hline Z & 0.166 & 0.039 \\
\hline
\end{tabular}

\section{DISCUSSION}

The key enabler of the collaborative system is the efficient integration of the technologies into the workers' daily usage.

- The modularity of the exoskeleton design allows the choice of different taskdependent modules. The trunk module is sufficient for stoop lifting of low weights. The passive arm modules provide constant body and load compensation, i.e., where the load remains in the hand of the worker for a longer period. For pick and place type activities, the active arm module is more appropriate as the lifting support can be adjusted continuously. For activities without bending, a simple backpack with the arms mounted would reduce the weight on the operator.

- In master-slave teleoperation, the Hydraulic Quadruped robot traverses the difficult terrain encountered by workers. The manipulator arm, with its integrated sensors and a dexterous end-effector, has anthropomorphic capabilities to perform complex tasks. For bilateral teleoperation, advanced control algorithms are used at the master station to maintain the closed-loop stability and transparency, mapping the kinematics, force feedback, as well as the control commands.

Specifically, for remote handling operations, the important criterion is the symbiotic integration of the individual technologies taking into account the communication media, the time-delay issues, and therefore, the closed-loop stability and transparency. Such a symbiotic integration is demonstrated through two instances:

1. The HyQ2Centaur [21]: With two robotic arms, the robot performs tasks like dooropening, valve-turning, and remote handling using its loco-manipulation capabilities, as seen in Fig. 9.

2. The HEXOTRAC system with a commercial manipulator arm (KUKA LWR) using the end-effector described in section 3.2.1.: At the master station, the vision-based OptiTrack motion capture system tracks the operator's wrist, while the fingertips are tracked in the HEXOTRAC linkages. The operator's wrist position and orientation is given as reference for controlling the manipulator's wrist in Cartesian space. The control of the end-effector hand with haptic feedback is performed through a novel Cartesian synergy-based tele-manipulation method described in [23], [24]. This bilateral interface enables intuitive and stable grasps irrespective of any master-slave asymmetry. Fig. 10 shows a sequence of images during the task.
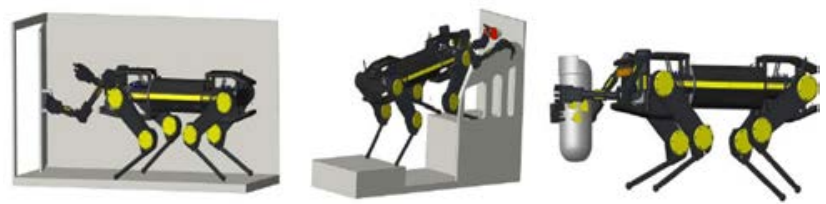

Figure 9: The task scenarios with the quadruped field robot, using loco-manipulation [21]. 


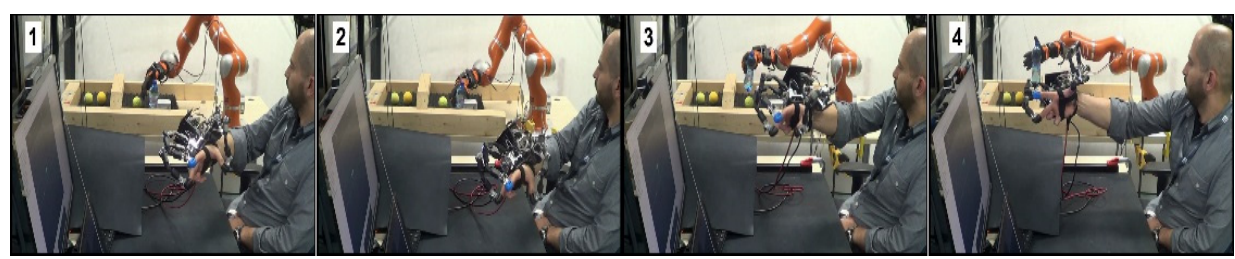

Figure 10: Teleoperation task with the HEXOTRAC master device and a robotic manipulator arm with end-effector as a slave device.

\section{CONCLUSIONS AND FUTURE WORK}

This article presented novel collaborative technologies dedicated to assisting and operating in industries, reducing the risks to the workers' health and safety. The technologies demonstrate the possibility of achieving significant step changes in state-of-the-art systems.

- The wearable, modular Robo-Mate exoskeleton presents significant reduction in muscle activity for the arm and lower back as well as related compression forces while bending or lifting.

- The Hydraulic Quadruped field robot provides a degree of stability and mobility that is crucial to its successful application in the hazardous and unstructured environments.

- The robotic manipulator hand with multiple degrees-of-freedom for extended reach and dexterity is capable of exerting: (i) maximum static grasp force (in power grasp) between 80 and $150 \mathrm{~N}$; (ii) maximum static grasp torque (in power grasp) between 2 and $5 \mathrm{Nm}$; and (iii) maximum static vertical lifting force of $160 \mathrm{~N}$.

- The hand exoskeleton with the key features of under-actuated design, easy wearand-remove, and unprecedented tracking accuracy, allows complete finger workspace, is suitable for a range of hand sizes, and allows full transparency without any hindrance to the finger flexion/extension, delivering rich perceptual cues.

The extension of this research continues the refinement of the technologies to investigate the impact on the usability in practice. Towards this goal, studies include the effect of the exoskeleton on the human body, the energy efficiency and autonomy of the quadruped field robot, the dexterity and feedback capabilities of the robotic manipulator arm, and haptic transparency and stability with the hand exoskeleton. In a later stage, various tests at enduser sites with operators in daily-use tasks are planned to further investigate the applicability and suitability of the systems.

\section{ACKNOWLEDGEMENT}

The research leading to these results shall be part of the project "Sistemi Cibernetici Collaborativi - Teleoperation \& Exoskeleton" funded under grant agreement with INAIL ("Istituto Nazionale per l'Assicurazione contro gli Infortuni sul Lavoro" - the Italian National Institute for Insurance against Workplace Injuries, CUP J32F17000950005).

\section{REFERENCES}

[1] Online sources: ILO Encyclopaedia. Online, http://www.iloencyclopaedia.org/part-ii44366/occupational-health-services. Accessed on: 17 May 2017. 
[2] Online sources: Sixth European Working Conditions Survey - Overview report: Executive summary. Online, https:/www.eurofound.europa.eu/sites/default/files/ ef_publication/field_ef_document/ef1634en1_0.pdf. Accessed on: 17 May 2017.

[3] Online sources: Estimating the cost of work-related accidents and ill-health: An analysis of European data sources. Online, https:/osha.europa.eu/sites/default/files/ publications/documents/Estimation_of_costs_of_work-

related\%20injuries_illnesses_deaths_\%20European_level.pdf.pdf. Accessed on: 17 May 2017.

[4] Online sources: Report - Work-related Low Back Disorders. Online, https://osha.europa.eu/sites/default/files/publications/documents/en/publications/repo rts/204/TE3200273ENC_-_Work-related_Low_Back_Disorders.pdf. Accessed on: 17 May 2017.

[5] McAlinden, J.J., Using robotics as an occupational health and safety control strategy. International Robot, 22(1), pp. 14-15, 1995.

[6] de Looze, M.P., Bosch, T., Krause, F., Stadler, K.S. \& O’Sullivan, L.W., Exoskeletons for industrial application and their potential effects on physical work load. Ergonomics, 59, pp. 671-681, 2015.

[7] Kaivo-Oja, J., A Review on the Future of Work: Robotics. EU-OSHA, 2015.

[8] Trevelyan, J.P., Kang S.C. \& Hamel, W.R., Robotics in Hazardous Applications. Springer Handbook of Robotics, Siciliano, B. \& Khatib, O. eds., pp. 1101-1126, 2008.

[9] Toxiri, S., Ortiz, J., Masood, J., Fernández, J., Mateos, L.A. \& Caldwell, D.G., A wearable device for reducing spinal loads during lifting tasks: Biomechanics and design concepts. Proceedings IEEE Intl. Conf. on Robotics and Biomimetics, pp. 2295-2300, 2015.

[10] Stadler, K. et al., Robo-mate an exoskeleton for industrial use-concept and mechanical design. Advances in Cooperative Robotics, 19, pp. 806-813, 2016.

[11] Semini, C., Tsagarakis, N.G., Guglielmino, E., Focchi, M., Cannella, F., \& Caldwell, D.G., Design of HyQ-A hydraulically and electrically actuated quadruped robot. Systems Control Engineering, 225, pp. 831-849, 2011.

[12] Semini, C. et al., Design of the Hydraulically-Actuated, Torque-Controlled Quadruped Robot HyQ2Max. IEEE/ASME Trans. On Mechatronics, 22(2), pp. 635-646, 2017.

[13] Gomis-Bellmunt, O. \& Campanile, L.F., Design Rules for Actuators in Active Mechanical Systems. Springer, 2010.

[14] Semini, C. et al., Additive manufacturing for agile legged robots with hydraulic actuation. Proceedings Intl. Conf. on Advanced Robotics, pp. 123-129, 2015.

[15] Negrello, F. et al., A modular compliant actuator for emerging high performance and fall-resilient humanoids. Proceedings IEEE-RAS Intl. Conf. on Humanoid Robots, pp. 414-420, 2015.

[16] Catalano, M.G., Grioli, G., Farnioli, E., Serio, A., Piazza, C. \& Bicchi, A., Adaptive synergies for the design and control of the Pisa/IIT softhand. International Journal of Robotics Research, 33, pp. 768-782, 2014.

[17] Sarakoglou, I., Brygo, A., Mazzanti, D., Hernandez, N.G. \& Caldwell, D.G., HEXOTRAC: A Highly Under-Actuated Hand Exoskeleton for Finger Tracking and Force Feedback. Proceedings IEEE Intl. Conf. on Intelligent Robots \& Systems, pp. 1033-1040, 2016.

[18] Barasuol, V., Buchli, J., Semini, C., Frigerio, M., Pieri, E.R.D. \& Caldwell, D.G., A reactive controller framework for quadrupedal locomotion on challenging terrain. Proceedings IEEE Intl. Conf. on Robotics and Automation, pp. 2554-2561, 2013. 
[19] Winkler, A. et al., Path planning with force-based foothold adaptation and virtual model control for torque controlled quadruped robots. Proceedings IEEE Intl. Conf. Robotics and Automation, pp. 6476-6482, 2014.

[20] Winkler, A., Mastalli, C., Havoutis, I., Focchi, M., Caldwell, D.G. \& Semini C., Planning and execution of dynamic whole-body locomotion for a hydraulic quadruped on challenging terrain. Proceedings IEEE Intl. Conf. on Robotics and Automation, pp. 5148-5154, 2015.

[21] Semini, C. et al., Design overview of the hydraulic quadruped robots HyQ2Max and HyQ2Centaur. Proceedings 14 ${ }^{\text {th }}$ Scandinavian Intl. Conf. on Fluid Power, 2015.

[22] Tsagarakis, N.G. et al., WALK-MAN: A High Performance Humanoid Platform for Realistic Environments. Journal of Field Robotics.

[23] Brygo, A. et al., Synergy-based interface for bilateral tele-manipulations of a masterslave system with large asymmetries. Proceedings IEEE Intl. Conf. in Robotics and Automation, pp. 4859-4865, 2016.

[24] Brygo, A. Sarakoglou, I., Grioli, G. \& Tsagarakis, N., Synergy-Based Bilateral Port: A Universal Control Module for Tele-Manipulation Frameworks Using Asymmetric Master-Slave Systems. Frontiers in Bioengineering and Biotechnology, 5(19), 2017. 